\title{
Spin orbit interaction and zitterbewegung in symmetric wells
}

\author{
Esmerindo Bernardes, ${ }^{1,}$ 月 John Schliemann, ${ }^{2,3}$ J. Carlos Egues, ${ }^{1,4,5}$, 周 and Daniel Loss ${ }^{3,5}$ \\ ${ }^{1}$ Instituto de Física de São Carlos \\ Universidade de São Paulo \\ Av. do Trabalhador São-carlense, 400 CP 369 \\ 13560.970 São Carlos, SP, Brazil \\ ${ }^{2}$ Institute for Theoretical Physics \\ University of Regensburg \\ D-93040 Regensburg, Germany \\ ${ }^{3}$ Department of Physics and Astronomy \\ University of Basel \\ CH-4056 Basel, Switzerland \\ ${ }^{4}$ Department of Physics and Astronomy \\ University of Basel \\ CH-4056 Basel, SwitzerlandK \\ ${ }^{5}$ Kavli Institute for Theoretical Physics \\ University of California, Santa Barbara, 93106 California, USA
}

(Dated: January 14, 2019)

\begin{abstract}
Recently, we have introduced a novel inter-subband-induced spin-orbit (s-o) coupling [Phys. Rev. Lett. 99, 076603 (2007); cond-mat/0607218] arising in symmetric wells with at least two subbands. This new s-o coupling gives rise to an usual zitterbewegung - i.e. the semiconductor analog to the relativistic trembling motion of electrons - with cycloidal motion without magnetic fields. Here we complement these findings by explicitly deriving expressions for the corresponding zitterbewegung in spin space.
\end{abstract}

\section{INTRODUCTION}

A controllable coupling between the spin and the orbital degrees of freedom in nanostructures is a highly desirable ingredient in the emerging fields of semiconductor spintronics and spin-based quantum computation and communication [1]. The gatetunable [⿰] confining potentials offers such a possibility. The seminal proposal of Datta and Das 四 of a spin field effect transistor highlights the use of the Rashba spin-orbit interaction to coherently rotate spins. More recently, the Rashba s-o interaction has been proposed as a convenient coherent mechanism for manipulating entangled electrons ("flying qubits") in the solid state [0]. Interestingly, systems with bulk inversion asymmetry exhibit an additional spin orbit interaction, termed the Dresselhaus s-o [5], whose interplay with the Rashba s-o can be used to devise a robust spin field effect transistor [0].

So far, s-o effects in 2DEGs have only been investigated in (asymmetric) quantum-well systems with a single confined state. Recently, we have introduced a new type of spin-orbit interaction present in quantum wells with two confined subbands [8]. Unlike the ordinary Rashba s-o interaction, ours is non-zero even in symmetric wells - as it arises from the inter-subbandcoupling between the lowest (even) and the first (odd) excited states of the well. As pointed out in Ref. [ह], this new intersubband-induced s-o coupling leads to an unusual dynamics of injected spin-polarized wave packets - the zitterbewegung or trembling motion - with cycloidal trajectories without magnetic fields. This is qualitatively different from the zitterbewegung in the presence of Rashba or Dresselhaus s-o interactions [G]. Here we complement the investigation in Ref. [8] by deriving explicit expressions for the zittebewegung in spin space, i.e., the spin dynamics of the injected electron. In what follows, we first present our new Hamiltonian with its eigenvalues and the corresponding time-evolution operator, and then proceed to determine the spin dynamics of injected electrons.

\section{EFFECTIVE HAMILTONIAN: NOVEL SPIN-ORBIT TERM}

Starting from the usual $8 \times 8$ Kane model one can derive a $2 \times 2$ effective Schrödinger equation for the conduction electrons by a folding down process. We have performed this procedure [8] for a symmetric quantum well with two subbands with edges at

\footnotetext{
*Electronic address: oousa@if.sc.usp.br
}

${ }^{\dagger}$ Electronic address: ggues@if.sc.usp.br 
the quantized energies $\varepsilon_{e}$ (lowest level) and $\varepsilon_{o}$ (first level). In terms of the real-spin $\sigma$ and pseudo-spin $\tau$ (describing the subband degree of freedom) Pauli matrices, our $4 \times 4$ Hamiltonian for an electrons with effective mass $m^{*}$ can be cast in the compact form

$$
\mathcal{H}=\left(\frac{p_{\|}^{2}}{2 m^{*}}+\epsilon_{+}\right) \mathbf{1} \otimes \mathbf{1}-\epsilon_{-} \tau^{z} \otimes \mathbf{1}+\frac{\eta}{\hbar} \tau^{x} \otimes\left(p_{x} \sigma^{y}-p_{y} \sigma^{x}\right), \quad \epsilon_{ \pm}=\frac{\varepsilon_{o} \pm \varepsilon_{e}}{2},
$$

where the new inter-subband-induced s-o coupling $\eta$ is given by

$$
\eta=-\left(\frac{1}{E_{g}^{2}}-\frac{1}{\left(E_{g}+\Delta\right)^{2}}\right) \frac{P^{2}}{3}\left\langle e\left|\partial_{z} V(z)\right| o\right\rangle+\left(\frac{\delta_{V}}{E_{g}^{2}}-\frac{\delta_{\Delta}}{\left(E_{g}+\Delta\right)^{2}}\right) \frac{P^{2}}{3}\left\langle e\left|\partial_{z} h(z)\right| o\right\rangle,
$$

where the Kane matrix element is $P=-i \hbar\left\langle S\left|p_{x}\right| X\right\rangle / m_{0}$, with $|S\rangle$ and $|X\rangle$ being the band-edge ( $\Gamma$ point) periodic Bloch functions, the fundamental and split-off band gaps in the well region are $E_{g}$ and $\Delta$, respectively, $|e\rangle$ and $|o\rangle$ denote the confined ground and first excited states of the well, $V(z)$ is the Hartree-type contribution to the potential, $h(z)$ is the profile function defining the quantum well, and $\delta_{v}, \delta_{\Delta}$ are the offsets between the valence bands in the well and barrier regions.

Note that $\mathcal{H}$ bears a close similarity to the usual Rashba Hamiltonian [\$]; however, the corresponding s-o coupling $\eta$ can be non-zero even for symmetric wells - this can be easily understood by noting that $\eta$ contains matrix elements between the ground and excited states. For a symmetric square-well potential, for instance, the first term in Eq. (2) vanishes (i.e. there is no Hartree contribution) and the second term reduces to

$$
\eta=\frac{2 P^{2}}{3}\left(\frac{\delta_{V}}{E_{g}^{2}}-\frac{\delta_{\Delta}}{\left(E_{g}+\Delta\right)^{2}}\right) \varphi_{o}(a) \varphi_{e}(a)
$$

where $\varphi_{i}(z) i=e, o$ denote the confined well wave functions. Note that $\partial_{z} h(z)=-\delta(z-a)+\delta(z-a)$ since $h(z)=\Theta(a-z)+\Theta(z-a)$ $[\Theta(z)$ : Heaviside function] defines a square well of width $L=2 a$. As shown in Ref. ([8]) the magnitude of $\eta$ can be comparable to that of the usual Rashba s-o coupling.

The Hamiltonian (1) can be easily diagonalized yielding the eigenvalues

$$
\mathcal{E}_{ \pm}=\varepsilon_{k} \pm \hbar \Omega
$$

where $\varepsilon_{k}=\frac{\hbar^{2} k_{\|}^{2}}{2 m^{*}}+\epsilon_{+}$and $\hbar \Omega=\sqrt{\epsilon_{-}^{2}+\eta^{2} k_{\|}^{2}}$.

\section{TIME EVOLUTION OPERATOR}

The time evolution operator $U=\exp (-i \mathcal{H} t / \hbar)$ can be straightforwardly obtained from Eq. (1). In the plane wave basis $\left\{\left|k_{11}, \sigma_{z}\right\rangle\right\}$, we find

$$
U=e^{-i \varepsilon_{k} t / \hbar}\left\{\cos (\Omega t) \mathbf{1} \otimes \mathbf{1}+i\left[\epsilon_{-} \tau^{z} \otimes \mathbf{1}-\eta \tau^{x} \otimes\left(k_{x} \sigma^{y}-k_{y} \sigma^{x}\right)\right] \frac{\sin (\Omega t)}{\hbar \Omega}\right\} .
$$

Next we use $U$ to calculate the time evolution of the spin operators.

\section{ZITTERBEWEGUNG IN SPIN SPACE}

To determine the spin dynamics in our system we have to calculate the time evolution of the tensor product $\mathbf{1} \otimes \sigma^{i}, i=x, y, z$. In the Heisenberg picture we have $\sigma_{H}^{i}(t)=U^{\dagger} \mathbf{1} \otimes \sigma^{i} U$, where $\sigma^{i}$, s denote the Pauli matrices at $t=0$. For the $z$ component we find

$$
\begin{gathered}
\sigma_{H}^{z}(t)=\left\{\cos (\Omega t) \mathbf{1} \otimes \mathbf{1}-i\left[\epsilon_{-} \tau^{z} \otimes \mathbf{1}-\eta \tau^{x} \otimes\left(k_{x} \sigma^{y}-k_{y} \sigma^{x}\right)\right] \frac{\sin (\Omega t)}{\hbar \Omega}\right\} \times \\
\mathbf{1} \otimes \sigma^{z}\left\{\cos (\Omega t) \mathbf{1} \otimes \mathbf{1}+i\left[\epsilon_{-} \tau^{z} \otimes \mathbf{1}-\eta \tau^{x} \otimes\left(k_{x} \sigma^{y}-k_{y} \sigma^{x}\right)\right] \frac{\sin (\Omega t)}{\hbar \Omega}\right\} \\
\sigma_{H}^{z}(t)=\mathbf{1} \otimes \sigma^{z}-\frac{\eta}{\hbar \Omega} \tau^{x} \otimes\left(k_{x} \sigma^{x}+k_{y} \sigma^{x}\right) \sin (2 \Omega t)-\frac{2}{\hbar^{2} \Omega^{2}}\left[\epsilon_{-} \eta \tau^{y} \otimes\left(k_{x} \sigma^{x}+k_{y} \sigma^{x}\right)+\eta^{2} k^{2}\right] \sin ^{2}(\Omega t) .
\end{gathered}
$$


Similarly, for the $x$ and $y$ components we obtain

$$
\begin{aligned}
& \sigma_{H}^{x}(t)=\mathbf{1} \otimes \sigma^{x}+\frac{\eta k_{x}}{\hbar \Omega} \tau^{x} \otimes \sigma^{z} \sin (2 \Omega t)+\frac{\eta k_{x}}{\hbar^{2} \Omega^{2}}\left[2 \epsilon_{-} \tau^{y} \otimes \sigma^{z}-\eta \mathbf{1} \otimes\left(k_{x} \sigma^{x}+k_{y} \sigma^{x}\right)\right] \sin ^{2}(\Omega t), \\
& \sigma_{H}^{y}(t)=\mathbf{1} \otimes \sigma^{y}+\frac{\eta k_{y}}{\hbar \Omega} \tau^{x} \otimes \sigma^{z} \sin (2 \Omega t)+\frac{\eta k_{y}}{\hbar^{2} \Omega^{2}}\left[2 \epsilon_{-} \tau^{y} \otimes \sigma^{z}-\eta \mathbf{1} \otimes\left(k_{x} \sigma^{x}+k_{y} \sigma^{x}\right)\right] \sin ^{2}(\Omega t) .
\end{aligned}
$$

In deriving the above equations we have used the algebraic relations $\left(\sigma^{i}\right)^{2}=\mathbf{1}, i=x, y, z$ and $\sigma^{i} \sigma^{j}=-\sigma^{j} \sigma^{i}=i \sigma^{k}, i, j, k=x, y, z$ (or any cyclic permutation), obeyed by the Pauli matrices. The $\tau^{i}, i=x, y, z$, also obey these relations. Equations (\$7)-(9) describe the spin dynamics of injected electrons due to the s-o coupling $\eta$. Together with the equations for $x_{H}(t)$ and $y_{H}(t)$ derived in Ref. [8], we have now the complete dynamics of an injected electron - i.e., the time dependence of both the spin and orbital degrees of freedom. As we discuss next, this dynamics are intrinsically linked due to the spin-orbit coupling and the fact that the $z$ component of the total angular momentum $\boldsymbol{j}$ is conserved.

\section{SPIN POLARIZED INJECTION AND DISCUSSION}

Similarly to Ref. [8] let us consider the case of a wide wave packet (approximated by a plane wave) initially injected into the lowest subband with spin up and an initial (group) velocity $\boldsymbol{v}_{g}=\hat{y} \hbar k_{0 y} / m^{*}$ along the $y$ axis. In this case we find from the above equations

$$
\begin{gathered}
\left\langle\sigma_{H}^{z}(t)\right\rangle=1-\frac{2 \eta^{2} k_{0 y}^{2}}{\hbar^{2} \Omega^{2}} \sin ^{2}(\Omega t), \\
\left\langle\sigma_{H}^{x}(t)\right\rangle=\left\langle\sigma_{H}^{y}(t)\right\rangle=0 .
\end{gathered}
$$

To better understand the above result we should recall that, as shown in Ref. [\$], the corresponding expectation values of $x_{H}(t)$ and $y_{H}(t)$ are

$$
\begin{aligned}
&\left\langle x_{H}(t)\right\rangle=\frac{\eta^{2} k_{0 y}}{(\hbar \Omega)^{2}} \sin ^{2}(\Omega t), \\
&\left\langle y_{H}(t)\right\rangle=\frac{\hbar k_{0 y}}{m^{*}} t+\frac{\eta^{2} k_{0 y} \epsilon_{-}}{2(\hbar \Omega)^{3}}[\sin (2 \Omega t)-2 \Omega t] .
\end{aligned}
$$

This oscillatory zitterbewegung shown in the above expectation values is proportional to the initial group wave length, a fact which offers way more favorable perspectives for the experimental detection of this effect than for free or weakly bound electrons. In the latter case the amplitude of the zitterbewegung is of order the free-electron Compton wave length and therefore by orders of magnitude smaller. For further details we refer to the discussion in Ref. [1]. Note also that differently from the zitterbewegung following from the ordinary Rashba s-o coupling in a single band [f], for which the trembling motion is perpendicular to the direction of propagation, here we find an oscillatory contribution in $\left\langle y_{H}(t)\right\rangle$ along the initial group velocity. From Eqs. (10) and (12) we can see that $\left\langle\sigma_{H}^{z}(t)\right\rangle=1-2 k_{0 y}\left\langle x_{H}(t)\right\rangle$. That is, the dynamics in spin and real spaces are coupled. To make this point even more explicit, let us calculate the time dependent expectation of the $z$ component of the orbital angular momentum: $l_{H}^{z}(t)=U^{\dagger} l^{z} \otimes \mathbf{1} U=U^{\dagger}\left(x p_{y}-y p_{x}\right) \otimes \mathbf{1} U$. A straightforward calculation yields

$$
\left\langle l_{H}^{z}(t)\right\rangle=\frac{\hbar}{2} \frac{2 \eta^{2} k_{0 y}^{2}}{\hbar^{2} \Omega^{2}} \sin ^{2}(\Omega t)=\hbar k_{0 y}\left\langle x_{H}(t)\right\rangle
$$

Since $s=\hbar \sigma / 2$ defines the spin angular momentum, we can immediately see that

$$
\left\langle s_{z H}(t)\right\rangle+\left\langle l_{z H}(t)\right\rangle=\hbar / 2
$$

The above result is easily undertood when we recall that at $t=0$ we injected a spin-up wave with zero angular momentum (i.e., at $t=0 j_{z}=s_{z}=\hbar / 2$ ). The conservation law in (15) is general (i.e., valid not just for the expectation values) and follows from the fact that $\left[l_{z}+s_{z}, \mathcal{H}\right]=0$. The constraint $\dot{l}_{z}=-\dot{s}_{z}$ together with the s-o coupling strongly tie the dynamics of the system thus leading to the zitterbewegung in both the spin and real variables. 


\section{SUMMARY}

In summary, we have derived expressions for the zitterbewegung in the spin variables due to the inter-subband-induced s-o coupling present in symmetric wells with two subbands. For the simple case of an injected spin-up plane wave, we explicitly show that the zitterbewegung in both the spin and real spaces are intrinsically linked. This follows from both the s-o coupling and the conservation of the $z$ component of the total angular momentum in our system.

\section{Acknowledgments}

The authors acknowledge useful discussions with S. Erlingsson, D. S. Saraga, M. Lee, D. Bulaev, J. Lehmann and M. Duckheim. This work was supported by the Swiss NSF, the NCCR Nanoscience, EU NoE MAGMANet, DARPA, ARO, ONR, JST ICORP, CNPq, FAPESP, and DFG via SFB 689.

[1] For an overview see e.g. Semiconductor Spintronics and Quantum Computation, eds. D. D. Awschalom, D. Loss, and N. Samarth, Springer, Berlin, 2002; I. Zutic, J. Fabian, and S. Das Sarma, Rev. Mod. Phys. 76, 323 (2004).

[2] G. Engels, J. Lange, Th. Schäpers, and H. Lüth, Phys. Rev. B 55, R1958 (1997); J. Nitta, T. Akazaki, H. Takayanagi, and T. Enoki, Phys. Rev. Lett. 78, 1335 (1997).

[3] E. I. Rashba, Fiz. Tverd. Tela (Leningrad) 2, 1224 (1960) (Sov. Phys. Solid State 2, 1109 (1960)); Y. A. Bychkov and E. I. Rashba, J. Phys. C 17, 6039 (1984).

[4] S. Datta and B. Das, J. Appl. Phys. 56, 655 (1990). See also J. C. Egues, G. Burkard, and D. Loss, Appl. Phys. Lett. 82 2658 (2003).

[5] G. Dresselhaus, Phys. Rev. 100, 580 (1955).

[6] J. C. Egues, G. Burkard, and D. Loss, Phys. Rev. Lett. 89, 176401 (2002)

[7] J. Schliemann, J. C. Egues, and D. Loss, Phys. Rev. Lett. 90, 146801 (2003).

[8] E. S. Bernardes, J. Schliemann, J. C. Egues and D. Loss, cond-mat/0607218.

[9] J. Schliemann, D. Loss, and R. M. Westervelt, Phys. Rev. Lett. 94, 206801 (2005); and Phys. Rev. B 73,085323 (2006). 